\title{
Eficacia de la asociación paracetamol-metamizol vs. paracetamol-dexketoprofeno en manejo de dolor agudo postoperatorio
}

\author{
M. García Ramiro, L. Alonso Guardo, A. Matilla Álvarez, R. Bartol Sevillano, L. M. Vaquero Roncero \\ y C. Muriel Villoria
}

Servicio de Anestesiología, Reanimación y Terapéutica del Dolor. Hospital Universitario de Salamanca. Salamanca

García Ramiro M, Alonso Guardo L, Matilla Álvarez A, Bartol Sevillano R, Vaquero Roncero LM, Muriel Villoria C. Eficacia de la asociación paracetamol-metamizol vs. paracetamol-dexketoprofeno en manejo de dolor agudo postoperatorio. Rev Soc Esp Dolor 2013; 20(6): 279-284.

\begin{abstract}
Objective: The use of drugs with different mechanisms, in combination for the treatment of pain, particularly acute postoperative pain, is a main part of the multimodal analgesia. The aim of this study was to evaluate the efficacy of metamizol plus paracetamol and compare it with the association of paracetamol plus dexketoprofen in acute postoperative pain.

Methods: We designed a prospective interventional study that included 42 patients undergoing general anesthesia for probably low algesic and duration of surgery < 120 minutes. All were treated with one grame of intraoperative paracetamol and then, in the resuscitation unit, when the score on the simple numeric scale was $>3$, they received the drug of the group that they were assigned (metamizol: group $\mathrm{M}$; dexketoprofen: group D), with evaluation of variations in the scale score. We examined in both groups demographic variables, variables related to anesthesia and surgery, simple numeric scale (NSE) changes, complications and the incidents during the process.

Results: 20 patients were studied in the M group and 22 patients in D group. Both groups had a similar distribution in terms of demographic variables, medical history, type and duration of surgery. Both group showed a clinically relevant decrease in the score of the simple numeric scale $(p<0.05)$. No differences were in the decrease of NSE when we compared the two groups together $(p>0.05)$.

Conclusion: Our results suggest that the combination of paracetamol plus metamizol in combined therapy for management
\end{abstract}

Recibido: 23-02-13

Aceptado: 01-03-13 of acute postoperative pain is effective and comparable to the combination of paracetamol plus dexketoprofen and can constitute an alternative therapy.

Key words: Paracetamol. Metamizol. Multimodal analgesia. Dexketoprofen. Acute postoperative pain.

\section{RESUMEN}

Objetivo: El uso de fármacos con mecanismos diferentes combinados entre sí para el tratamiento del dolor, en concreto del dolor agudo postoperatorio, forma parte fundamental de un tipo de analgesia llamada multimodal. El objetivo de este trabajo es evaluar la eficacia de la asociación de paracetamol más metamizol y compararla con la asociación de paracetamol más dexketoprofeno en dolor agudo postoperatorio.

Métodos: Diseñamos un estudio prospectivo de intervención en el que se incluyeron 42 pacientes sometidos bajo anestesia general a cirugías de probable bajo nivel algésico y duración < 120 minutos. Todos ellos fueron tratados con un gramo de paracetamol intraoperatorio $y$, posteriormente en reanimación, cuando la puntuación en la escala numérica simple fue mayor de 3, recibieron el fármaco del grupo al que fueron asignados (metamizol: grupo $\mathrm{M}$; dexketoprofeno: grupo D), evaluando las variaciones en la puntuación de dicha escala. Se examinaron en ambos grupos las variables demográficas, las variables relacionadas con la anestesia, aquellas relacionadas con la cirugía, las variaciones en la escala numérica simple (ENS), la aparición de complicaciones y las incidencias durante el proceso.

Resultados: 20 pacientes fueron estudiados en el grupo M y 22 pacientes en el grupo D. Los dos grupos siguieron una distribución similar en cuanto a variables demográficas, antecedentes médicos, tipo y duración de la cirugía a la que se sometieron. En ambos grupos se observó una disminución clínicamente relevante en la puntuación de la escala numérica simple $(\mathrm{p}<0,05)$. Sin embargo, no existieron diferencias en la disminución de ENS al comparar los dos grupos entre sí ( $p>0,05)$. 
Conclusión: Nuestros resultados sugieren que la combinación de paracetamol y metamizol como terapia combinada para el manejo de dolor agudo postoperatorio es eficaz y equiparable a la combinación de paracetamol y dexketoprofeno, pudiendo constituir, por tanto, una alternativa a esta última.

Palabras clave: Paracetamol. Metamizol. Analgesia multimodal. Dexketoprofeno. Dolor agudo postoperatorio.

\section{INTRODUCCIÓN}

El manejo del dolor agudo postoperatorio es un tema de gran importancia para los anestesiólogos (1). Es un fenómeno complejo y multifactorial que implica el conocimiento de polimorfismos genéticos y vías analgésicas, que sugieren que la terapia óptima debe ir encaminada a tratar cada tipo de dolor con aquellos fármacos que posean un mecanismo de acción apropiado, con el menor número de efectos adversos (1).

A esto hace referencia el concepto de analgesia multimodal, a la combinación de fármacos con mecanismos de acción diferentes, que producen un aumento de la potencia analgésica, sin aumentar los efectos adversos o toxicidad (2).

En los últimos 20 años, los analgésicos no opioides han ganado importancia en el tratamiento del dolor agudo postoperatorio como componentes de dicho tipo de analgesia (3).

De entre ellos, el paracetamol o acetaminofén ha llegado a ser el fármaco más usado como primera línea de tratamiento en fiebre y dolor (4), y su preparación intravenosa ha producido un aumento considerable de su uso en el periodo perioperatorio por parte de los anestesiólogos. Su mecanismo de acción es aún incierto, pero parece claro que carece de actividad sobre COX periféricamente, y que posee una acción central, lo que le confiere un mecanismo de acción diferente al de los AINE (1-6).

Otro de los fármacos ampliamente utilizado es el metamizol o dipirona. Su mecanismo de acción continúa siendo incierto también, pero se postula una actuación a nivel central como inhibidor de la isoenzima $\operatorname{COX} 3$, diferente al del paracetamol (3).

En este sentido, motivados por el concepto de analgesia multimodal, diversos estudios evalúan la variación en la calidad analgésica postoperatoria tras combinar analgésicos (metamizol o paracetamol) y AINE, frente al uso individual de cada uno de ellos (7-10).

Pero por otro lado, la evaluación en terapia multimodal de la combinación paracetamol-metamizol para la mejora del dolor agudo, no se refleja con frecuencia en la bibliografía.
Así, y puesto que son escasos los trabajos que miden la eficacia de la combinación paracetamol y metamizol, diseñamos este estudio con el objetivo de evaluar si ambos fármacos utilizados en combinación en el tratamiento perioperatorio de pacientes sometidos a cirugías de duración $<120$ minutos y bajo nivel algésico son eficaces, evaluando las diferencias al compararlo con la combinación de paracetamol y AINE, ya demostrada eficaz (7-10).

\section{MATERIAL Y MÉTODOS}

Realizamos un estudio prospectivo de intervención durante un periodo de cinco meses en el Hospital Clínico de Salamanca, previa consulta con el Comité Ético. Se incluyeron 42 pacientes mayores de 16 años, sometidos a cirugía (urgente o programada) bajo anestesia general, de duración $<120$ minutos y con una intensidad de dolor postoperatorio previsiblemente baja.

Pacientes menores de 16 años, sometidos a anestesia diferente a anestesia general, en tratamiento crónico con cualquier tipo de analgesia, uso de cloruro mórfico perioperatorio y analgesia intraoperatoria con algún analgésico no opioide diferente a paracetamol, fueron los criterios de exclusión.

Tras la inclusión en el estudio y la firma del consentimiento informado, los pacientes fueron distribuidos aleatoriamente en dos grupos: grupo $\mathrm{M}$ de 20 pacientes (grupo metamizol) y grupo D de 22 pacientes (dexketoprofeno). Tras anestesia general, todos los pacientes incluidos en el estudio recibieron una dosis intraoperatoria de 1 gramo de paracetamol intravenoso, 20 minutos antes del final de la cirugía. Posteriormente, durante su estancia en reanimación, se evaluó la escala numérica simple (ENS) cada 10 minutos, recibiendo 2 gramos intravenosos de metamizol (grupo M) o 50 miligramos de dexketoprofeno (grupo D), cuando la valoración de ENS era $>3$.

Las variables a estudio fueron: demográficas (edad, sexo, peso, talla, IMC), antecedentes médicos y quirúrgicos, tratamiento habitual, tipo y duración de la cirugía, dosis de inducción y mantenimiento de hipnóticos, relajantes musculares y opioides.

Fueron recogidos los tiempos desde la administración de la última dosis de opioide hasta la administración del paracetamol durante la cirugía, así como el momento de la administración del paracetamol, y la diferencia en tiempo, entre el momento de la puesta de paracetamol y la del tratamiento correspondiente al grupo de estudio.

El dolor fue medido mediante la ENS tanto al ingreso en reanimación, como previo a la administración de metamizol o dexketoprofeno, y a los 20 minutos tras el tratamiento. Recogimos todas las complicaciones o incidencias atribuidas por los investigadores a los fármacos. 
Calculamos el tamaño muestral necesario para una diferencia clínicamente relevante en la ENS de 3 puntos entre ambos grupos, con una desviación estándar (DS) de 3 y un error alfa de 0,05 , siendo necesarios para encontrar esta diferencia al menos 17 pacientes en cada grupo.

Las variables de estudio se expresan en medias, desviaciones estándar, medianas, rangos intercuartílicos y porcentajes. Los test estadísticos utilizados fueron U de Mann Whitney y el test de Wilcoxon para variables pareadas, asumiendo un nivel de significación $\mathrm{p}<0,05$. El programa utilizado para los cálculos fue SPSS $® 12.0$.

\section{RESULTADOS}

Analizamos una muestra total de 42 pacientes ( 20 en el grupo M y 22 en el grupo D).

En la tabla I podemos ver la distribución de las variables demográficas en los dos grupos.

TABLA I. DATOS DEMOGRÁFICOS Y CARACTERÍSTICAS GENERALES

\begin{tabular}{lcc}
\hline Variables & Grupo $M(n=20)$ & Grupo $D(n=22)$ \\
\hline Edad & $52(34,25-62,75)$ & $34,50(25,75-52,00)$ \\
Peso & $162(160-170)$ & $166,50(159-175)$ \\
Talla & $69,25(59,25-79,25)$ & $69,50(61-78,50)$ \\
IMC & $25,25(23,17-27,22)$ & $24,40(22,23-26,03)$ \\
\hline
\end{tabular}

Datos expresados en medianas y rangos intercuartílicos.

Respecto al sexo, el $50 \%$ de hombres fueron incluidos en el grupo M, y $50 \%$ en el grupo D. En cuanto a los antecedentes médicos, la mayoría de pacientes fueron ASA I y II, suponiendo un $89 \%$ del total de la muestra.

En el grupo M, el $35 \%$ se trataba de cirugía endoscópica nasosinusal (CENS), un $25 \%$ tumorectomías por neoplasia de mama, un $20 \%$ fueron apendicectomías por laparotomía, un $15 \%$ consistieron en avulsión por cordales incluidos, y el $5 \%$ restante cirugías inespecíficas. La media de la duración de estas intervenciones fue de 75,75 (DS 34,65) minutos.
En los pacientes tratados con dexketoprofeno, el 36,4\% se sometieron a avulsión por cordales incluidos (cirugía maxilofacial), un 22,7 \% fueron intervenidos de apendicitis aguda, otro $22,7 \%$ a CENS, y el 18,12\% restante fueron mujeres intervenidas por cáncer de mama. La duración media en este caso fue de 81,36 (DS 29,77) minutos.

Se recogieron los tiempos desde la administración de la última dosis de opioide hasta la de paracetamol y del analgésico del grupo de estudio, así como el tiempo que transcurrió desde la administración de paracetamol hasta la administración del fármaco correspondiente para cada grupo (Tabla II).

Las dosis de hipnóticos, opioides y relajantes musculares empleadas en la inducción y mantenimiento de la anestesia están reflejadas en la tabla III.

La ENS a su llegada a Reanimación fue de mediana 1 (0-4) para el grupo M y de $1(0-3)$ para el grupo D $(p<$ $0,05)$.

La ENS previa al inicio del tratamiento fue de 5 (4-6) en el grupo M, siendo un $35 \%$ de los valores superiores a 5. En el grupo D, la mediana de ENS fue de 5 (4-6), con un $33 \%$ de los casos superior a 5 .

El tiempo, en minutos, transcurrido desde su llegada a Reanimación hasta la administración del tratamiento fue comparable en ambos grupos, siendo en el grupo D una mediana de 7 y para el grupo M de 15 .

Tras administrar el tratamiento con metamizol o dexketoprofeno se reevaluó la ENS a los 20 minutos. Los resultados los podemos observar en la tabla IV.

La reducción de la ENS en cada grupo, evaluada previa a la administración del fármaco y a los 20 minutos posteriores, fue de 3 (2-4) en el grupo M, y de 3 (2-4) en el grupo $\mathrm{D}(\mathrm{p}<0,05)$.

Durante el seguimiento, ningún paciente presentó efectos adversos relacionados con el tratamiento ni otras complicaciones.

\section{DISCUSIÓN}

El objetivo del estudio era observar si existían diferencias en cuanto a la calidad analgésica de dos terapias con fármacos no opioides con diferentes mecanismos de acción. Los resultados indican que no existen diferencias

TABLA II. TIEMPOS DE ADMINISTRACIÓN DE ANALGÉSICOS DURANTE LA CIRUGÍA Y POSTOPERATORIOS

\begin{tabular}{lccc}
\hline & $\begin{array}{c}\text { Inicio de paracetamol tras } \\
\text { administración última dosis } \\
\text { de opioide }\end{array}$ & $\begin{array}{c}\text { Inicio tratamiento desde } \\
\text { administración de } \\
\text { paracetamol }\end{array}$ & $\begin{array}{c}\text { Inicio tratamiento desde } \\
\text { administración de última } \\
\text { dosis de opioide }\end{array}$ \\
\hline Metamizol (min) & $7,5(13,75-28,75)$ & $45(30-63,75)$ & $60(36-101,25)$ \\
Desketoprofeno (min) & $2,5(10-11,25)$ & $37(30-51,25)$ & $45(28,75-51,25)$ \\
Significación $(\mathrm{p})$ & $0,479 *$ & $0,470 *$ & $0,091 *$ \\
\hline
\end{tabular}

Datos expresados en medianas. *Sin diferencias estadísticamente significativas entre ambos grupos $(p>0,05)$. 
TABLA III. FÁRMACOS EMPLEADOS DURANTE EL ACTO ANESTÉSICO

\begin{tabular}{|c|c|c|c|}
\hline \multicolumn{2}{|c|}{ Grupo metamizol $(n=20)$} & \multicolumn{2}{|c|}{ Grupo dexketoprofeno $(n=22)$} \\
\hline Hipnótico inducción & Propofol $=20$ & Hipnótico inducción & Propofol $=22$ \\
\hline Dosis hipnótico inducción (mg) & $180(150-200)$ & Dosis hipnótico inducción (mg) & $175(147,5-200)$ \\
\hline Hipnótico mantenimiento & $\begin{array}{l}\text { Propofol }=15 \\
\text { Sevorane }=5\end{array}$ & Hipnótico mantenimiento & $\begin{array}{l}\text { Propofol }=16 \\
\text { Sevorane }=6\end{array}$ \\
\hline $\begin{array}{l}\text { Dosis hipnótico mantenimiento } \\
\text { (mg) }\end{array}$ & $450(359-587)$ & $\begin{array}{l}\text { Dosis hipnótico mantenimiento } \\
(\mathrm{mg})\end{array}$ & $432(400-540)$ \\
\hline Relajante muscular inducción & $\begin{array}{c}\text { Succinilcolina }=5 \\
\text { Rocuronio }=8 \\
\text { Cisatracurio }=4 \\
\text { Ninguno }=3\end{array}$ & Relajante muscular inducción & $\begin{array}{c}\text { Succinilcolina }=7 \\
\text { Rocuronio }=11 \\
\text { Cisatracurio }=2 \\
\text { Ninguno }=2\end{array}$ \\
\hline $\begin{array}{l}\text { Dosis relajante muscular inducción } \\
(\mathrm{mg})\end{array}$ & $42,50(10-50)$ & $\begin{array}{l}\text { Dosis relajante muscular inducción } \\
(\mathrm{mg})\end{array}$ & $50(30-100)$ \\
\hline $\begin{array}{l}\text { Dosis relajante muscular } \\
\text { mantenimiento (mg) }\end{array}$ & 0 & $\begin{array}{l}\text { Dosis relajante muscular } \\
\text { mantenimiento }(\mathrm{mg})\end{array}$ & $0(36-25)$ \\
\hline Opioide inducción & $\begin{array}{c}\text { Fentanilo }=18 \\
\text { Remifentanilo }=3\end{array}$ & Opioide inducción & $\begin{array}{c}\text { Fentanilo }=18 \\
\text { Remifentanilo }=4\end{array}$ \\
\hline Dosis opioide inducción (mcg) & $150(150-162,50)$ & Dosis opioide inducción $(\mathrm{mcg})$ & $150(100-150)$ \\
\hline Opioide mantenimiento & $\begin{array}{c}\text { Fentanilo }=6 \\
\text { Remifentanilo }=14\end{array}$ & Opioide mantenimiento & $\begin{array}{l}\text { Fentanilo }=15 \\
\text { Remifentanilo }=7\end{array}$ \\
\hline Dosis opioide mantenimiento $(\mathrm{mcg})$ & $275(150-320)$ & Dosis opioide mantenimiento $(\mathrm{mcg})$ & $150(150-250)$ \\
\hline
\end{tabular}

TABLA IV. VARIACIÓN DE LA MEDIANA DE LAS ENS PARA CADA GRUPO EN REANIMACIÓN, CUANDO EL ENS FUE SUPERIOR A 3, Y A LOS 20 MINUTOS POSTERIORES A LA ADMINISTRACIÓN DE ANALGESIA CON DEXKETOPROFENO O METAMIZOL

\begin{tabular}{lccc}
\hline & ENS pre tratamiento & ENS 20 min postratamiento & Significación ENS pre/post \\
\hline Grupo M & $5(4-6)$ & $2(1-2)$ & $\mathrm{p}<0,05^{* *}$ \\
Grupo D & $5(4-6)$ & $2(1-2,5)$ & $\mathrm{p}<0,05^{* *}$ \\
Significación entre grupos & $\mathrm{p}>0,05^{*}$ & $\mathrm{p}>0,05^{*}$ & \\
\hline
\end{tabular}

*Sin diferencias significativas entre grupos. **Diferencias significativas en reducción de ENS dentro de cada grupo.

significativas en la calidad analgésica ni en la reducción del dolor, al comparar ambos regímenes.

Las cirugías elegidas, en tiempo y características de los pacientes (predominio de ASA I y II), son similares en ambos grupos y comparables a las evaluadas en otros estudios existentes en la literatura, como en el caso de Sniezeck y cols. (11), que evaluaron distintos regímenes analgésicos en cirugía de Mohs o micrográfica, de bajo nivel algésico. Por su parte, Aspley y cols. (12) evaluaron la analgesia en cirugía dental, con un dolor de similar intensidad al produci- do en las cirugías de nuestro trabajo, al igual que el estudio de Mehlisch y cols. (13). En el trabajo de Brodner y cols. (14) compararon distintas terapias analgésicas en cirugías menores e intermedias. Mitchell y cols. (15) lo realizaron sobre colecistectomías laparoscópicas y herniorrafias, consideradas de nivel algésico intermedio. Otros estudios en otro tipo de cirugías con dolor postoperatorio de similares características serían el de Clark y cols. (16) en cirugía pediátrica; Hiller y cols. (17) y Korkmaz y cols. (18) en cirugía traumatológica; y Kamondetdecha y cols. (19) en cirugía obstétrica. 
Nuestros resultados en cuanto a la mejoría en ENS en el grupo tratado con paracetamol y AINE, son similares a los obtenidos en todos estos trabajos citados anteriormente, al igual que en otros como en el de Moore y cols. (20), Daniels y cols. (21) y Mehlisch y cols. (13). En todos ellos concluyen que la combinación de analgésicos en estos modelos de dolor agudo postoperatorio es una herramienta eficaz y muestran una reducción en la puntuación de las escalas clínicamente importante.

Dos revisiones sistemáticas -Romsing y cols. (7) y Ong y cols. (8)- avalan también estos resultados.

En cuanto a las características de las pautas analgésicas, las empleadas en nuestro estudio son comparables a las utilizadas en estudios previos $(11,20,22-25)$.

Tanto la elección del mórfico intraoperatorio (fentanilo), como las dosis del mismo que podrían condicionar tanto el grado de analgesia postoperatoria como el momento del rescate, son muy parecidos en ambos grupos y sin diferencias significativas (Tabla III).

El tiempo transcurrido entre la última dosis del mórfico y la utilización del paracetamol de control son también comparables entre ambos grupos, anulando otro de los factores que podría condicionar una diferencia en la escala ENS de los pacientes a su llegada a Reanimación, y por tanto su comparabilidad.

Además, el tiempo hasta la utilización de la terapia de rescate es casi idéntico en los dos grupos, permitiendo una mejor comparación de la eficacia analgésica de los fármacos en estudio.

Por otro lado, la reducción observada en la puntuación de ENS en nuestro trabajo, en el grupo paracetamol más dexketoprofeno, la encontramos en estudios previos, donde la disminución oscila entre un 20-40 \% $(22,23,26,27)$.

También encontramos esta mejoría analgésica en el grupo paracetamol más metamizol (sin diferencias significativas con respecto al grupo de dexketoprofeno), y si bien el metamizol ha demostrado su eficacia en la disminución del dolor agudo postoperatorio en otros estudios de forma aislada $(28,29)$, o en combinación con AINE $(30,31)$, desconocíamos su eficacia analgésica combinado con paracetamol en el tratamiento del dolor postoperatorio.

Esta mejoría analgésica se podría explicar por la combinación de fármacos con distintos mecanismos de acción con el fin de producir un sinergismo, sumando sus efectos, sin aumentar los efectos adversos de los mismos (2).

Aunque la distribución de las cirugías elegidas es similar en ambos grupos, esta podría constituir una limitación al mismo, al no ser los grupos perfectamente comparables con respecto al umbral algésico. Por otro lado, la no utilización de un grupo placebo o de un fármaco aislado como control, puede condicionar las comparaciones, si bien esto lo hemos obviado por motivos éticos. En cuanto al tiempo de seguimiento (20 minutos desde la administración del fármaco en estudio), puede ser algo reducido, si bien la disminución en la ENS en ambos grupos llega hasta ENS $<3$ (umbral que se considera como clínicamente aceptable) (32), sin precisar otros tratamientos. El uso de mórfico no lo recogimos, pero llegando a estos niveles de ENS, es muy probable que no fuera necesaria su utilización, aunque es cierto que puede limitar la validez de nuestros resultados.

\section{CONCLUSIÓN}

Los analgésicos no opioides tienen un papel muy importante en el manejo del dolor agudo postoperatorio medio y moderado. En dolor severo son un componente fundamental de la analgesia multimodal, disminuyendo el consumo de opioides.

La asociación de paracetamol y metamizol es eficaz en la reducción del dolor y en el aumento de la eficacia analgésica, y supone una alternativa eficaz a la combinación de paracetamol y AINE.

No obstante, sería conveniente realizar estudios de mayor envergadura con el fin de obtener resultados más concluyentes.

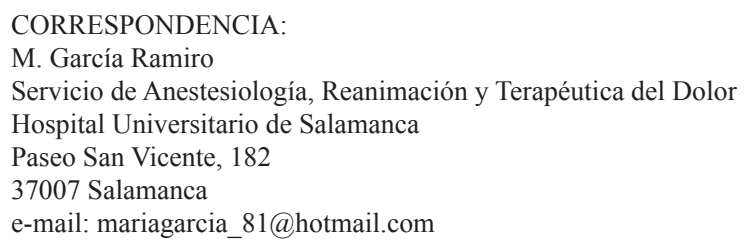

\section{BIBLIOGRAFÍA}

1. Toussaint K, Yang XC, Zielinski MA, Reigle KL, Sacavage SD, Nagar D, et al. What do we (not) know about how paracetamol (acetaminophen) works? Journal of Clinical Pharmacy and Therapeutics 2010;35:617-38.

2. Joshi GP. Técnicas de analgesia multimodal y de rehabilitación postoperatoria. Anesthesiology Clin N Am 2005;23:185202.

3. Schug SA, Manopas A. Uptodate on the role of non-opioids for postoperative pain treatment. Best Practice \& Research Clinical Anaesthesiology 2007;21:15-30.

4. Mattia C, Coluzzi F. What anesthesiologists should know about paracetamol (acetaminophen). Minerva Anestesiol 2009;75:664-53.

5. Oscier CD, Milner QJW. Peri-operative use of paracetamol. Anaesthesia 2009;64:65-72.

6. Schug SA, Garrett WR, Gillespie G. Opioid and non-opioids analgesics. Best Practice \& Research Clinical Anaesthesiology 2003;17:91-110.

7. Romsing J, Moiniche S, Dahl JB. Rectal and parenteral paracetamol, and paracetamol in combination with NSAIDs, for postoperative analgesia. Br J Anesthesia 2002;88:215-26. 
8. Ong CK, Seymour RA, Lirk P, Merry AF. Combining paracetamol (acetaminophen) with nonsteroidal antiinflammatory drugs: A qualitative systematic review of analgesic efficacy for acute postoperative pain. Anesth Analg 2010;110(4):1170.

9. Remy C, Marret E, Bonnet F. Effects of acetaminophen on morphine side-effects and consumption after major surgery: Meta-analysis of randomized controller trials. Br J Anesth 2005;94:505-13.

10. Elia N, Lysakowski C, Tramer MR. Does multimodal analgesia with acetaminophen, nonesteroidal antiinflamatory drugs, or selective cyclooxygenase- 2 inhibitors and patient-controlled analgesia morphine offer advantages over morphine alone? Anesthesiology 2005;103:1269-73.

11. Sniezek PJ, Brodlan DG, Zitelli JA. A randomized controlled trial comparing acetaminophen, acetaminophen and ibuprofen, and acetaminophen and codeine for postoperative pain relief after Mohs surgery and cutaneous reconstruction. Dermatol Surg 2011;37:1007-13.

12. Aspley S, Mehlisch DR, Daniels SE, Southerden KA, Christensen KS. A single tablet combination of ibuprofen/paracetamol in the management of moderate to severe postoperative dental pain: a multicenter, two-stage, randomized, double-blind, parallel-group, placebo-controlled, factorial study. World Congress Pain; 2010 Abstract PW 385.

13. Mehlisch DR, Aspley S, Daniels SE, Bandy DP. Comparison of the analgesic efficacy of concurrent ibuprofen and paracetamol with ibuprofen or paracetamol alone in the management of moderate-to-severe postoperative dental pain. World Congress Pain; 2010 Abstract PW 384.

14. Brodner G, Gogarten W, Van Aken H, Hahnenkamp K, Wempe C, Freise H, et al. Efficacy of intravenous paracetamol compared to dipyrone and parecoxib for postoperative pain management after minor-to-intermediate surgery: a randomised, double-blind trial. Eur J Anaesthesiol 2011;28(2):125-32.

15. Mitchell A, Van Zanten SV, Inglis K, Porter G. A randomized controlled trial comparing acetaminophen plus ibuprofen versus acetaminophen plus codeine plus caffeine after outpatient general surgery. J Am Coll Surg 2008;206:472-9.

16. Clark E, Plint AC, Correll R, Gaboury I, et al. A randomized controlled trial of acetaminophen, ibuprofen, and codeine for acute pain relief in children with musculoskeletal trauma. Pediatrics 2007;119:460-7.

17. Hiller A, Silvanto M, Savolainen S, Tarkkila P. Propaceta$\mathrm{mol}$ and diclofenac alone and in combination for analgesia after elective tonsillectomy. Acta Anaesthesiol Scand 2004;48:1185-9.

18. Korkmaz Dilmen O, Tunali Y, Cakmakkaya OS, Yentur E, Tutuncu AC, Tureci E, et al. Efficacy of intravenous paracetamol, metamizol and lornoxicam on postoperative pain and morphine consumption after lumbar disc surgery. Eur J Anaesthesiol 2010;27(5):428-32.

19. Kamondetdecha R, Tannirandorn Y. Ibuprofen versus acetaminophen for the relief of perineal pain after childbirth: A randomized controlled trial. J Med Assoc Thai 2008;91:282-6.

20. Moore RA, Straube S, Paine J, Derry S, McQuay HJ. Minimum efficacy criteria for comparisons between treatments using individual patient meta-analysis of acute pain trials:
Examples of etoricoxib, paracetamol, ibuprofen, and ibuprofen/paracetamol combinations after third molar extraction. Pain 2011;152:982-9.

21. Daniels SE, Aspley S, Reader S, Mehlisch DR, Southerden KA. A comparison of the analgesic efficacy and tolerability of a combination of ibuprofen $400 \mathrm{mg}$ plus paracetamol 1000 $\mathrm{mg}$, a combination of ibuprofen $200 \mathrm{mg}$ plus paracetamol $500 \mathrm{mg}$, a combination of ibuprofen $400 \mathrm{mg}$ plus codeine $25.6 \mathrm{mg}$, a combination of paracetamol $1000 \mathrm{mg}$ plus codeine $30 \mathrm{mg}$, and placebo in postoperative dental pain following third molar extraction. World Congress Pain; 2010 Abstract PH 382.

22. Breivik EK, Barkvoll P, Skovlund E. Combining diclofenac with acetaminophen or acetaminophen-codeine after oral surgery: A randomized, double-blind single-dose study. Clin Pharmacol Ther 1999;66:625-35.

23. Dahl V, Dybvik T, Steen T, Aune AK, Rosenlund EK, Raeder JC. Ibuprofen vs. acetaminophen vs. ibuprofen and acetaminophen after arthroscopically assisted anterior cruciate ligament reconstruction. Eur J Anaesthesiol 2004;21:471-5.

24. Ianiro SR, Jeansonne BG, McNeal SF, Eleazer PD. The effect of preoperative acetaminophen or a combination of acetaminophen and ibuprofen on the success of inferior alveolar nerve block for teeth with irreversible pulpitis. J Endod 2007;33:11-4.

25. Doherty M, Hawkey C, Goulder M, et al. A randomised controlled trial of ibuprofen, paracetamol or a combination tablet of ibuprofen/paracetamol in community-derived people with knee pain. Ann Rheum Dis 2011;70:1534-41.

26. Gazal G, Mackie IC. A comparison of paracetamol, ibuprofen or their combination for pain relief following extractions in children under general anaesthesia: A randomized controlled trial. Int J Paediatr Dent 2007;17:169-77.

27. Montgomery JE, Sutherland CJ, Kestin IG, Sneyd JR. Morphine consumption in patients receiving rectal paracetamol and diclofenac alone or in combination. Br J Anaesth 1996;77:445-7.

28. Edwards JE, Meseguer F, Faura CC, Moore RA, McQuay HJ. Single-dose dipyrone for acute postoperative pain. Cochrane Database of Systematic Reviews 2001;(3):CD003227.

29. Steffen P, Schuhmacher I, Weichel T, Georgieff M, Seeling W. Differential administration of non-opioids in postoperative analgesia, I. Quantification of the analgesic effect of metamizole using patient-controlled analgesia. Anasthesiologie, Intensivmedizin, Notfallmedizin, Schmerztherapie 1996;31(3):143-7.

30. Oberhofer D, Skok J \& Nesek-Adam V. Intravenous ketoprofen in postoperative pain treatment after major abdominal surgery. World Journal of Surgery 2005;29(4):446-9.

31. Samulak D, Michalska M, Gaca M, Wilczak M, Mojs E, Chuchracki M. Efficiency of postoperative pain management after gynecologic oncological surgeries with the use of morphine + acetaminophen + ketoprofen versus morphine + metamizol + ketoprofen. Eur J Gynaecol Oncol 2011;32(2):168-70.

32. Allvin R, Brasseur L, Crul B, Dhal J, Lehmann K, Nicosia F, et al. European Minimum standards for the management of postoperative pain. EuroPain Task Force. Pegasus Helthcare Intl, UK; 1998. 